\title{
Admission avoidance and early discharge of acute hospital admissions: an accident and emergency based scheme
}

\author{
C Hardy, D Whitwell, B Sarsfield, C Maimaris
}

\begin{abstract}
Objectives-To validate an accident and emergency (A\&E) based approach to assisting early discharge or avoiding admission to acute hospital beds by means of two separate teams, one in hospital and the other in the community, working closely together at the interface between primary and secondary health care.

Design-A purpose designed admission avoidance (AA) team was established in the $A \& E$ department, and a target group of patients identified whose admissions might be avoided or curtailed. A rapid response community team (RRCT) based in Cambridge was also established to provide basic health care to patients in their homes after discharge from hospital. The key elements of the project were rapid assessment, careful selection of patients, early decision making at senior level, and
\end{abstract} close liaison with the community team.

Results-During the first year (1999) of the project the AA team assessed 785 patients and 257 patients were eventually discharged home to the care of the RRCT. Of these, 149 patients (58\%) were comparable to a historical control group (from 1997/98), with regard to their demographic and clinical characteristics and care needs, and had an average length of hospital stay of 1.7 days compared with 6.3 days for the control group. The remaining 108 patients were not directly comparable but were supported by the teams because the benefits were clear and exclusion would have been unethical. These patients had an average length of stay of seven days. The readmission rate was 3 of $257(1.2 \%)$ for the intervention group and 8 of $531(1.5 \%)$ for the control group. A limited patient satisfaction survey among patients cared for at home revealed that $97 \%$ of patients were "satisfied to very satisfied" with the care provided. The RRCT had also looked after an additional 194 patients from other sources (total $=451)$, including postoperative orthopaedic early discharges from an adjacent hospital. The average length of care at home by the RRCT for all 451 patients was 6.6 days. The annual cost of the two teams was $£ 113900$.

Conclusions-These results indicate that an $A \& E$ based approach to the identification of patients suitable for short-term domiciliary support that aims rapidly to restore previous levels of independence, can reduce the burden of acute admissions to hospital without reducing quality of care or patient satisfaction. The scheme has now been established on a permanent basis and extension of this strategy to other patient groups is under evaluation. (Emerg Med f 2001;18:435-441)

Keywords: hospital admission; hospital discharge

There has been widespread acknowledgement in recent years of the growth in emergency hospital admissions, ${ }^{1}$ an increase of $50 \%$ over the 13 years before 1994 in Scottish hospitals ${ }^{2}$ being one example. Over a six year period from 1993 to 1999, at Addenbrooke's hospital in Cambridge, the increase was $27 \%$ in all adult emergency admissions, and $76 \%$ increase in adult admissions via the accident and emergency (A\&E) department. Inpatient bed numbers have not increased and pressure has therefore mounted on hospitals to accelerate turnover and reduce admission rates. The recent National Bed Enquiry by the Department of Health has found that "there has been a fall in the number of hospital beds in England over the last 40 years that has left the NHS unable to cope with health care needs". The report recommends an increase of 4000 beds in England over the next five years, 2000 in acute and general beds and 2000 in intermediate care. ${ }^{3}$

One approach nationally has been to set up "hospital at home" schemes, for example those reviewed by Illiffe in a $B M \mathcal{F}$ overview in June $1998^{4}$ in Northamptonshire ${ }^{5}$ and Bristol. ${ }^{6}$ Similarly, a recent trial in Leicester compared patient care in "hospital at home" with that in hospital. ${ }^{7}$ The economic evaluation found fewer days of care in the hospital at home. ${ }^{89}$ There seems little doubt that these schemes generally have a successful health outcome compared with inpatient care and are preferred by patients. ${ }^{5-8}$ Their size and case mix have been similar though not always directly comparable, but descriptions of the cultural changes entailed in especially the secondary care centres, have not been widely aired and do have implications for service developments in the future.

Another approach has been to discharge elderly patients at high risk of readmission to the care of a home treatment team lead by a district nurse for six weeks or less. ${ }^{10}$ This patient group had disabling medical conditions including Parkinson's disease, arthritis, 
dementia and cerebrovascular disease. The results were fewer re-admissions at six weeks and a year and may have important implications because of the high proportion of re-admissions among all emergency admissions.

This paper will describe another approach: a target group of patients, who would normally require acute hospital admission would be identified early on by an A\&E department based team. These patients would have relatively short-term rehabilitation needs, which would be met at home by a designated community team. The hypothesis is that their length of hospital stay could be reduced or the admission avoided altogether. Also that basing the team in an $\mathrm{A} \& \mathrm{E}$ department is a logical and efficient approach because of key elements of rapid assessments, careful selection of patients, early decision making at senior level, and interface with a specially designed community support team.

\section{Methods}

A project team (CH, DW, CM) based in the A\&E department, analysed baseline data to identify groups of patients who by virtue of their clinical characteristics might be able to avoid admission altogether or be admitted for short periods only and then discharged to their homes with support.

The data analysed covered a year period (October 1997 to September 1998) and included all patients admitted via $\mathrm{A} \& \mathrm{E}$ department. Of the 56785 patients attending the $\mathrm{A} \& \mathrm{E}$ department during this time $11419(20.1 \%)$ were admitted to wards under various specialties. The average length of stay for each specialty was obtained and varied from 13 days for department of medicine for the elderly patients, nine days for general medicine, eight days for trauma, to two for plastic surgery and 1.5 days for patients admitted to the A\&E department observation ward. Several groups of patients were identified who seemed to be uncomplicated cases and therefore amenable to an accelerated discharge pathway. Of the 1560 patients admitted with trauma not requiring operative treatment, 531 patients required minimal medical treatment but needed analgesia, nursing care and mobilisation, as well as a detailed assessment of their needs on discharge. These 531 patients had sustained upper and/or lower limb trauma such as fractures or severe soft tissue contusions. Though these conditions varied in detail from patient to patient, they formed a simple group with broadly similar needs. Many of them were elderly and were admitted either under trauma or department of medicine for the elderley. The demographic details, clinical characteristics, care needs and average length of hospital stay was obtained. Seventy one per cent were female and the age range was $<65$ years $12 \%$, $65-75$ years $17 \%$, and $>75$ years $71 \%$. This group of patients was considered to be most suitable for the project and formed a retrospective control group.

The target patients were those in the same diagnostic categories between January 1999 and December 1999, and formed the interven-

\section{Box 1 Criteria for selection and inclusion into the admission avoidance and early discharge project}

- Patient would normally be admitted to acute orthopaedic or department of medicine for the elderly bed.

- Aged 16 years and over.

- Upper or lower limb trauma not requiring operative treatment or further investigation for underlying acute medical condition.

- If needed, occupational therapy/physiotherapy assessments are completed before discharge.

- Independent with transfers: bed/chair/ wheelchair/commode (if living alone).

- Resident in Cambridge, South Cambridgeshire or East Cambridgeshire.

- Not resident in nursing home.

- Registered with a GP.

- Access to phone at discharge destination.

- Need for nursing/therapy support to meet care plan.

- The perceived period of rehabilitation will not exceed an average of two weeks.

tion group. Comparison was made between the historical control and the intervention group. The outcome measures were the length of hospital stay and readmission rates. For the intervention group a limited patient satisfaction survey was carried out.

The pilot project started in January 1999. Though this did not match exactly the months of the control group, both groups span over a year period, so that any seasonal bias was removed. The health authority and the hospital trust supported the scheme. The hospital and community teams were set up and time was allowed for training and education of all the NHS staff that were helping to identify possible patients.

A clinical assistant (3.5 hours daily) and a nurse ( 7.5 hours daily) six days a week (Monday to Saturday) based in the A\&E department of Addenbrooke's Hospital identified suitable patients according to a set of criteria (see box 1). Patients were recruited from the $A \& E$ department or among those admitted to the observation, department of medicine for the elderly or trauma wards. The patients' medical needs were met promptly. Fast track occupational therapy (OT) and physiotherapy assessments, where appropriate, were arranged. Patients thus assessed and found to be still suitable were sent home to the support of the rapid response community team (RRCT), sponsored by the Cambridge-based Lifespan Community Trust. This consisted of a district nurse coordinator who took referrals, organised visits and monitored patient progress, supported by five part time (30 hours a week) health care assistants. The team was able to provide assistance with hygiene and dressing as well as giving medication, rehabilitation, mobilisation and simple exercises. They quickly developed close links with community therapists who supported them when needed. Care 


\section{Box 2 Characteristics of patients assessed by the $A \& E$ Admission} Avoidance (AA) team for consideration for the project

BREAKDOWN OF 785 PATIENTS ASSESSED BY THE HOSPITAL TEAM INTO DIFFERENT CATEGORIES

66 Patients had care packages in place prior to admission. These were reviewed in light of current needs and restarted when appropriate to enable early discharge.

90 Patients did not live in the area covered by the community team so it was not possible to avoid admission.

238 Patients were refereed for assessment but did not fulfil the criteria.

133 Patients were assessed but found that did not require community support.

257 Patients were discharged to the care of the community team.

1 Patient could not be discharged because the community team caseload was full.

was given for up to two weeks. Patients were then either discharged or transferred to social service support. Outpatient follow up was organised before leaving hospital in the usual way. General practitioners (GP) were informed of the discharges by fax and became involved where necessary (only in a few cases during the pilot). District nurses were asked to visit project patients within 24 hours of discharge from hospital so as to initiate any involvement in the care plan and to establish a senior level of nursing supervision.

As the project progressed, it became evident that there was extra capacity in the RRCT and patients from other diagnostic groups and clinical characteristics began to be assessed and discharged home under the care of the

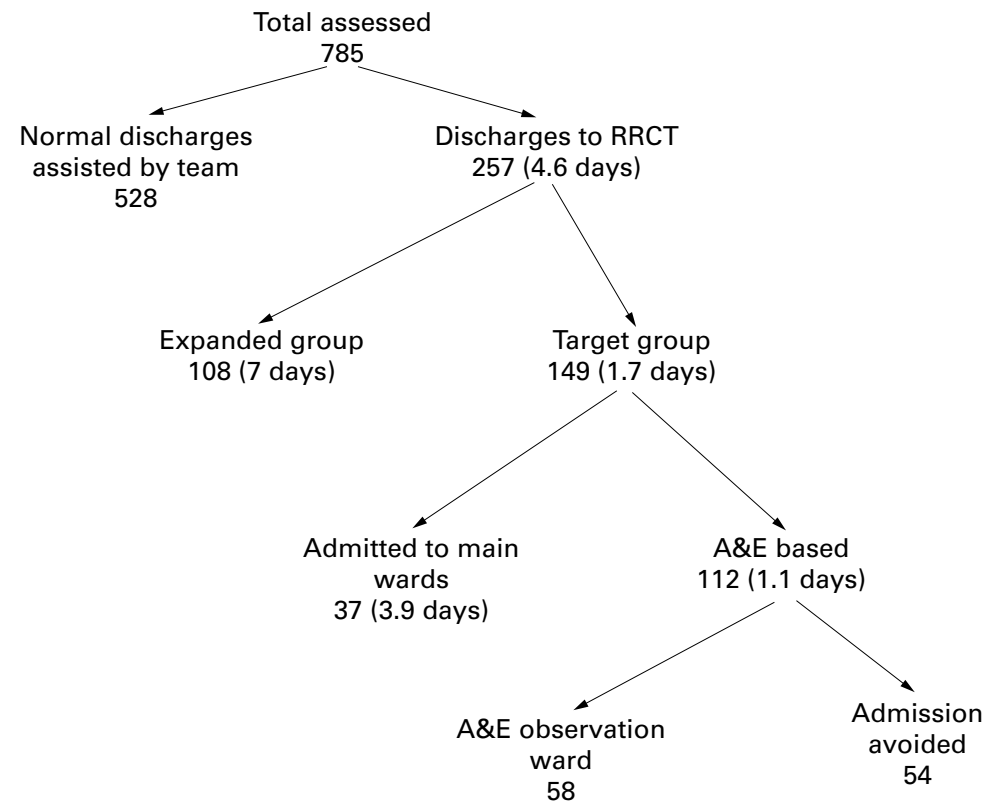

Figure 1 Outcome of patient assessments by AA team (average length of stay in parentheses).
RRCT. Although these patients spanned a wider group of diagnoses (cardiac failure, diabetes, pneumonia, chronic obstructive airways disease, cellulitis needing intravenous antibiotics), they still had to meet the original selection criteria (see box) and had to have simple rehabilitation needs with a prospective rehabilitation period of two weeks or less. Also, postoperative elective hip and knee replacement patients began to be included in the caseload of the community team from both Addenbrooke's and the Hinchinbrooke Hospitals (for patients resident in the area covered by the community trust), though these were not part of the intervention group.

Interim evaluations of the project were carried out at three and six months. The hospital evaluation was based on length of hospital stay and readmission rates and compared the intervention group with the control group. For the community aspects of care, a limited patient satisfaction survey was carried out, measures being based on adequacy of analgesia, understanding of explanations given, and satisfaction of care in hospital and in the community. The patient compliance was 32 of 117 $(34 \%)$. At six months the health authorities decided to continue the project for a whole year. This study reports the results for the whole year of the project.

\section{Results}

In 1999, 11031 of 57054 (19.3\%) patients attending the $\mathrm{A} \& \mathrm{E}$ department were admitted to the hospital. The $\mathrm{A} \& \mathrm{E}$ department based admission avoidance (AA) team assessed 785 patients $(7.1 \%$ of $\mathrm{A} \& \mathrm{E}$ department admissions), of whom 257 were discharged to the care of the RRCT. The 785 patients were grouped into broad categories as shown in box 2. These assessments represent a considerable amount of time, which would not have been available from any other hospital staff group. Liaison between the hospital and community teams became a vital and highly effective part of successful early discharges and admission avoidances. The demographic characteristics of the 257 patients, cared for at home by the RRCT, are as follows: women $=76 \%$, age range: $<65$ years $11 \%, 65-75$ years $13 \%,>75$ years $76 \%$. A detailed breakdown of the 257 patients is shown in figure 1 .

Our intention was to compare the patients discharged to the RRCT with a similarly sized historical control group. The control group was established using hospital records, selecting patients according to the initial criteria for intervention - that is, upper and or lower limb trauma such as fractures/dislocations needing manipulation and cast immobilisation and rehabilitation, chest wall or pubic rami fractures treated conservatively or moderately severe contusions needing elevation and rehabilitation. As mentioned above, the criteria for treatment were extended as the study progressed, including additional patients with wider diagnostic categories (to use the community team to as near full capacity as possible). Of the final 257 patients in the treated group, $149(58 \%)$ met the initial inclusion criteria, 
and were therefore directly comparable with the pre-project historical control group; these 149 formed the intervention group. The looser entry criteria of the remaining 108 patients made this group more heterogenous and complex, rendering comparison invalid; they were not included in the comparative analysis of the two groups.

Individual matching of patients between the intervention and control groups was not carried out, but the demographic characteristics of the two groups (age, sex, place of residence), the clinical characteristics (diagnosis, treatment, comorbidities) and the social and personal needs were similar. The average length of stay of the intervention group was 1.7 days compared with 6.3 days for the control group. This represents a significant reduction of 4.6 bed days per patient or a total of 685 hospital bed days for the 149 patients of the intervention group.

In 1999,3 of the $257(1.2 \%)$ patients were readmitted to hospital: one because of psychiatric problems (unrelated to her fall) and one because of pressure from relatives. One patient required re-manipulation of a fracture as an inpatient (this was unrelated to care provided by the teams) but continued under the care of the RRCT until discharge. The readmission rate for the control group was 8 of $531(1.5 \%)$.

Thirty two patients out of 117 (34\%) discharged to the RRCT completed the patient satisfaction survey carried out in the first six months of the project. Of these, $97 \%$ were very satisfied to satisfied with their discharge arrangements from hospital. Satisfaction with analgesia was not applicable in every case, because pain was not always a feature, but $60 \%$ of all patients felt that pain relief was good to satisfactory on arrival home. Finally, 97\% were very satisfied to satisfied with their care by the RRCT and none were dissatisfied.

An evaluation of the community team activities was also carried out. In 1999 the RRCT cared for a total of 451 patients divided into the following groups: $257(57 \%)$ patients from the A\&E department team, $35(8 \%)$ patients directly from the community (GPs and practice nurses) that would have required hospital admission if the RRCT was not available, 22 $(5 \%)$ patients with cellulitis requiring intravenous antibiotics and 137 (30\%) postoperative orthopaedic departmentic patients. The average length of care at home by the RRCT of all 451 patients was 6.6 days: $54 \%$ up to one week, $43 \%$ up to two weeks and $3 \%$ over two weeks. After discharge by the community team $36 \%$ of the 257 patients gained full independence, $24 \%$ continued with their existing small care package, $20 \%$ were given ongoing care by district nurses for a further period and $20 \%$ were assessed for new short-term care packages.

The postoperative total hip and knee replacement patients were discharged home from hospital to the RRCT between day 4-7 after the operation and were kept under the care of the RRCT until the 14th postoperative day. The average reduction of hospital stay for this group was estimated to be around six days but a detailed evaluation of this group was outside the scope of this study.

cosTs

The pilot project used some existing resources, namely senior medical, nursing and other support staff and input at the hospital and in the community. Extra funding however was made available by the health authorities to establish the two teams. The hospital based team consisting of the clinical assistant (3.5 hours daily for five days) and nurse (7.5 hours daily, six days a week, Monday to Saturday) cost $£ 52400$ per annum and was paid by the Cambridgeshire Health Authority. The community team-district nurse coordinator and five part time ( 30 hours a week) health care assistantscost £61500 and was paid by Lifespan Community NHS Trust. The total cost was $£ 113900$ per annum for a total of 451 patients cared for in the community.

We tried to calculate the cost for the inpatient care of our patients. The finance department at Addenbrooke's hospital estimate that the average general cost (variable and semi-fixed only-not including capital charges or medical time expenses) of an occupied bed for similar patients is around $£ 170$ per day. We therefore estimate that for the intervention group alone (149 patients), the cost savings would be around £116450.

\section{Discussion}

In this study we have shown that an $A \& E$ based team can successfully effect early discharge of selected patients and achieve their rehabilitation by short-term community support, to an acceptable level of satisfaction for the patients and with a worth while saving of hospital bed days. The results of this pilot project have been achieved by the establishment of the two teams and the close working relationships between them at the interface of primary and secondary care. The acute trusts and health authorities have been fully supportive of the project and agreed to make available the financial resources for the setting up of the two teams. The senior staff at Addenbrooke's Hospital and the A\&E department provided guidance, leadership and support of the scheme.

This is a comparative study using a historical control group. We were only able to accurately compare 149 of 257 patients recruited by the hospital team with our control group and these patients formed our intervention group. It limited the number of patients recruited to the intervention group but we are confident that in this way, the two groups are strictly comparable (demographic and clinical characteristics).

The main outcome measure was the length of hospital stay and we have shown that this is reduced by 4.6 bed days per patient or a total of 685 bed days. As such, this has proved the hypothesis formulated at the beginning of the study correct. The extension to include other patients so as to maximise the use of the community team as a service provider and to justify the financial input of the various contributors, expanded the boundaries of the initial pilot project and for this reason we cannot 
accurately state the number of bed days saved in total. However, the 22 patients needing intravenous antibiotics and 35 other patients from the community would have required hospital admission. The average length of stay would probably be determined according to the specialty they would normally be admitted under (department of medicine for the elderly 13 , medicine 9 , trauma 8 , plastic surgery 1.5 and $A \& E$ department observation 1.3 days). It has also been estimated but not accurately determined that the average length of stay for the 137 postoperative orthopaedic patients was reduced by around six days (total of 822 bed days).

Department of medicine for the elderly beds in the local community have been substantially reduced in recent years, but the number of acute beds for this section of the population did not increase proportionately. Patients awaiting type 3 accommodation currently block a substantial proportion of department of medicine for the elderly and orthopaedic beds. As the majority of patients requiring support after discharge were elderly, the impact of this project was likely to be greatest on these beds, because of the diagnostic categories of the target group. The needs of suitable patients had to be simple and a working guideline of referral to the AA team within 48 hours of admission was used, if patients were referred from the wards. Patients who had been in the hospital longer than this tended to have developed more complex medical, nursing and social needs. With a prospective rehabilitation period of two weeks or less, selection of patients had to be rigorous. The evaluation showed $97 \%$ of patients had been discharged within that two weeks, and $60 \%$ achieved their former level of independence in that time while another $20 \%$ managed with some extra support from district nurses.

The majority of patients were entered into the project directly from the $\mathrm{A} \& \mathrm{E}$ department and many needed short-term admission to the $\mathrm{A} \& \mathrm{E}$ observation ward. The A\&E observation ward proved to be an excellent base for rapid assessments by physiotherapists and OT, and the frequency of review of patients by senior staff meant that progress could be followed up and decisions amended accordingly, facilitating the early discharges. Also, the nursing and other staff are used to rapid turnover and have multiple mechanisms in place to achieve that end which constitutes a difference of emphasis from other departments of the hospital. The cost of observation ward beds is at present slightly less than that of other wards.

In the PROFET study of 1996, A\&E departments are taken to task for underrecording functional disability caused by falls and under-using support services. ${ }^{11}$ Many of the patients in our control and intervention groups were elderly patients who had had falls and we felt that the OT and physiotherapy assessments of the hospital phase and the design of the RRCT in the community phase answered this criticism. Also that these elements were supported by referral to other agencies: the GP concerned or referral to the local "fallers" clinic, run by the department of medicine for the elderly. The department covers investigation of medical causes for falls, including iatrogenic (polypharmacy) as well as other causes related to advanced age (failing vision, stability, etc) and also offers physiotherapy services to restore mobility and confidence.

The project expanded to include other groups of patients because it was felt that a critical mass of patients was necessary to make the community team both financially viable and able to maintain the composition and experience. We believe that it is relevant to describe all these other groups of patients and include them in this paper as we are describing a different approach to the NHS problem of limited hospital bed resources. This problem becomes especially acute during the winter months and the description of this approach and the results in this study are particularly relevant.

The patient satisfaction survey was limited to the first six months of the study and the response rate of $34 \%$ was low. While only limited conclusions may therefore be drawn the feedback received indicated that the patients seemed to be satisfied with the service provided. We acknowledge that this is a subjective outcome measure and the sample was small but the comments received from the patients were extremely encouraging and complimentary: "Care in A\&E superb". "Treated with great kindness". "Stressful waiting for the morning visit when not sure exact time (varied between 8-11 am). Overall a very good service".

The overall readmission rate of $1.2 \%$ is similar to that for the control group. This proves that the scheme is effective in keeping patients in their own home and effectively rehabilitating them to their previous levels of independence. Some patients needed new social care packages, which were initiated by the community team. This put pressure on the social services to ensure they were able to take up any ongoing care needs.

The hospitals benefited by the reduction of length of stay. For the intervention group (149 patients) alone, we estimate that the hospital would have saved at least $£ 116400$ on general costs. However, because of the increasing pressures, both from acute admissions and elective work, the hospitals used the bed days saved by the scheme for other purposes. Thus the project has allowed the hospitals to improve their efficiency and their bed capacity. In the arguments that continue about whether "hospitals at home" schemes provide a supplement or a substitute for existing services, we would conclude that ours provides a combination that is difficult to quantify, in common with other studies. The costs for care of patients, whose admissions were avoided or reduced, have partly been shifted towards the primary care sector. We also believe there were indirect benefits in changing the culture and attitude of hospital staff, especially in the wards as a result of the introduction of the scheme. The project has highlighted to many staff that it is possible 
to aim to continue the hospital care in the community for a limited period for a selected group of patients. We would have saved the hospital more bed days if we could discharge the 90 patients (see box 2), suitable for the scheme from other counties (Suffolk, Essex and Hertfordshire) if they were able to provide such a service too.

We were able to set up this project at an extra cost of $£ 113900$, because we used existing systems and senior medical input from the A\&E department; OT and physiotherapy from within the hospital and some other community resources from the intermediate care teams. We are unable to quantify the cost element of the work carried out by these other staff for this particular project. We can state, however, that the extra cost to the health authorities for the 451 patients (looked after in the community on average 6.6 days) was $£ 250$ per patient. This included not only the care provided in the community but also the hospital assessments. This seems to be an extremely efficient system because it builds on existing services ${ }^{12}$ and patients remain in their own homes. The scheme therefore could be reproduced in other parts of the country relatively quickly, thereby contributing to tackling the shortage of beds in the NHS, highlighted by the National Bed Enquiry of the Department of Health.

In conclusion, these results were obtained with a focused patient group. They provide proof of principle that a coordinated approach to the identification of patients suitable for rapid or immediate discharge, and to continuing domiciliary support and management can significantly relieve the burden of admissions to hospital without reducing quality of care or patient satisfaction. Basing this process in an $\mathrm{A} \& \mathrm{E}$ department forms a natural extension of its function and is highly successful.

We thank the Cambridgeshire Health Authority, Addenbrooke's NHS Trust and Lifespan Community NHS Trust for supporting the pilot study and the continuation of the project. We also thank all those who participated and worked for the project and collected data and Kathie Butcher for supervision. Thanks to Rosemary Rooks and Jill Briggs for designing and setting up the community team and to Chris Sice for helping with data collection.

Contributors

Charlotte Hardy collaborated in designing the pilot, coordinating the study, analysing the data and helped in writing the paper. Debbie Whitwell participated in the design and coordination of the study and collection and analysis of data. Beryl Sarsfield coordinated the community team and participated in collection and evaluation of data. Chris Maimaris directed the project, collaborated in the design and coordination of the study, participated in analysing and interpreting the data and helped in writing the paper. Chris Maimaris and Charlotte Hardy are the guarantors of the paper.

Funding: the authors received no funding for the research and the writing of the paper. The Cambridgeshire Health Authority funds the project.

Conflicts of interest: none.

1 Hobbs R. Rising emergency admissions [see comments]. BMF 1995;310:207-8.

2 Kendrick $S$. The pattern of increase in emergency hospital admissions in Scotland. Health Bull Edinb 1996;54:169-83.

3 Department of health. Shaping the future NHS: long term planning for hospitals and related services. Consultation planning for hospitals and related services. Consultation of health, 2000. Also in BMF 2000;320:463 "Government of health, 2000. Also in BMF 2000;320:4

4 Iliffe S. Hospital at home: from red to amber. Data that will liffe $\mathrm{S}$. Hospital at home: from red to amber. Data that will
reassure advocates-but without satisfying the sceptics. $B M \mathcal{F}$ 1998;316:1761-2

5 Shepperd S, Harwood D, Gray A, et al. Randomised controlled trial comparing hospital at home care with inpatient hospital care. I: three month follow up of health outcomes. BMF 1998;316:1786-91.

6 Richards HS, Coast J, et al. Randomised controlled trial comparing effectiveness and acceptability of an early discharge, hospital at home scheme with acute hospital care. BMF 1998;316:1791-6.

7 Wilson A, Parker H, Wynn A, et al. Randomised controlled trial of effectiveness of Leicester hospital at home scheme with hospital care. BMF 1999;319:1542-6.

8 Jones J, Wilson A, Parker H, et al. Economic evaluation of hospital at home versus hospital care: cost minimisation analysis of data from randomised controlled trial. BMf analysis of data from

9 Shepperd S, Harwood D, Gray A, et al. Randomised controlled trial comparing hospital at home care with inpaient hospital care. II: cost minimisation analysis. $B M \mathcal{F}$ 1998; 316:1791-6

10 Martin F, Oyewole A, Moloney A. A randomized controlled trial of a high support, hospital discharge team for elderly people. Age Ageing 1994;23:228-34.

11 Close J, Ellis M, Hooper R, et al. Prevention of falls in the elderly trial (PROFET): a randomised controlled trial. Lancet 1999;353:93-7.

12 Shepperd S. Report on community diversion schemes. Cambridge: NHS Executive Eastern Region Report, 1999. 